\title{
Adsorption of fibronectin derived from serum and from human endothelial cells onto tissue culture polystyrene
}

\author{
P. B. van Wachem, B. W. L. Mallens, A. Dekker, T. Beugeling, J. Feijen, A. Bantjes, \\ J.P. Detmers, ${ }^{*}$ and W. G. van Aken \\ Department of Chemical Technology, Twente University of Technology, P.O. Box 217, \\ 7500 AE Enschede, The Netherlands; ${ }^{*}$ Hospital Ziekenzorg, Enschede, The Netherlands
}

Human endothelial cells (HEC) suspended in a culture medium containing $20 \%$ human serum (CMS) adhere and spread on(to) moderately wettable polymers, such as tissue culture polystyrene (TCPS). We have previously shown that serum derivedfibronectin, which is a cell adhesion promoting protein, has a high affinity for TCPS, but that the amount of fibronectin which adsorbed from CMS was relatively small. In this study we investigated whether fibronectin derived from HEC contributes to the adhesion and spreading of the cells on(to) TCPS. Therefore, HEC were seeded in the presence of fibronectindepleted CMS. The amount of fibronectin detected on TCPS increased with both cell seeding density and incubation time. Although initial HEC adhesion is delayed on TCPS which had been precoated with albumin (Alb), high density lipoprotein (HDL) or immunoglobulin G (IgG), maximal numbers of adhering and spreading HEC were found on these surfaces $6 \mathrm{~h}$ after seeding of HEC. Fibronectin was detected on these surfaces, but an exchange of preadsorbed Alb, HDL, or IgG for fibronectin could not be demonstrated. We conclude that HEC deposit fibronectin onto TCPS, irrespective of the presence of a preadsorbed layer of proteins which delay cell adhesion.

\section{INTRODUCTION}

In the presence of culture medium containing $20 \%$ serum (CMS), human endothelial cells (HEC) adhere, spread and proliferate on(to) moderately wettable tissue culture polystyrene (TCPS). ${ }^{1}$ Cell adhesion was reduced or absent when more hydrophilic or more hydrophobic polymers were used. ${ }^{1}$ During the adhesion process of HEC, serum protein adsorption occurs, which depends on the surface properties of the polymer. Besides the composition of the adsorbed serum protein layer, the conformation of adsorbed proteins ${ }^{2}$ and/or protein adsorption/desorption and exchange processes on TCPS-like surfaces ${ }^{3}$ may be important for HEC adhesion.

Experiments in which polymeric surfaces were precoated with albumin (Alb), high-density lipoprotein (HDL), immunoglobulin G (IgG), and fibronectin $(\mathrm{Fn})$ showed that Alb, HDL and IgG inhibit or delay the adhesion 
of HEC while Fn promotes HEC adhesion. ${ }^{4}$ When we studied the adsorption of proteins from CMS onto TCPS and other polymers, a relatively small amount of Fn was detected upon TCPS but not on more hydrophobic surfaces. ${ }^{4}$ In addition, Alb, HDL, and to a lesser extent IgG were also present on TCPS.

We have suggested that deposition of cellular-derived Fn (cFn) may also contribute to the adhesion, spreading and proliferation of HEC on(to) TCPS, ${ }^{4}$ possibly by an exchange of adsorbed serum proteins such as Alb, HDL, and/or IgG for $\mathrm{CFn}$. The likelihood of exchange of adsorbed protein for serum and/or cellular Fn was indirectly supported by our findings that precoating of TCPS with Alb, HDL or IgG only delayed the adhesion of HEC during the first hour after cell seeding: after $6 \mathrm{~h}$ the numbers of HEC which adhered and spread on(to) uncoated and precoated TCPS were about the same. ${ }^{4}$ The exchange of surface bound protein for Fn may also be deduced from experiments carried out by Grinnell. ${ }^{5} \mathrm{He}$ found that more serum-Fn adsorbed onto TCPS precoated with native Alb, than onto TCPS precoated with denatured Alb.

These observations prompted further investigation on the role of $\mathrm{Fn}$ in relation to HEC adhesion, in particular, the contribution of $\mathrm{cFn}$ in the adhesion, spreading and proliferation of HEC on(to) TCPS. Furthermore, the possible exchange of Alb, HDL and IgG, preadsorbed onto TCPS, for (cellular) Fn was studied.

\section{MATERIALS AND METHODS}

\section{Cell culture}

Endothelial cells were isolated from human umbilical cord veins according to the isolation method originally given by Jaffe et al. ${ }^{6}$ with some modifications. ${ }^{7}$

Cells were routinely cultured in tissue-culture polystyrene (TCPS) flasks (Corning, NY, USA) precoated with a partially purified fibronectin $\left(\mathrm{Fn}^{\mathrm{c}}=\right.$ crude fibronectin, which was obtained from the Central Laboratory of the Netherlands Red Cross Blood Transfusion Service (CLB), Amsterdam, The Netherlands, and which is a coproduct from the cryoprecipitate preparation of coagulation factor VIII, $2 \%$ solution $(\mathrm{w} / \mathrm{v})$ in "complete medium," see below).

Serum-containing culture medium (CMS) consisted of complete medium, which is an equal mixture of Medium M 199 and Medium RPMI 1640 (both from Gibco Biocult Co., Paisley, U.K.), with $2 \mathrm{mM}$-L-glutamine (Merck, Darmstadt, (W.G.), $100 \mathrm{U} / \mathrm{mL}$ penicillin, $100 \mu \mathrm{g} / \mathrm{mL}$ streptomycin (both from Flow Lab., Irvine, U.K.) and $2.5 \mu \mathrm{g} / \mathrm{mL}$ fungizone (Gibco), which was supplemented with $20 \%$ human serum (pool from 20 healthy male donors). Cell cultures were not screened for mycoplasma. 
Cells were used in experiments after the second or third passage when cultures had reached confluence.

\section{Cell incubation and lysis}

TCPS (24 wells clusters) was obtained from Costar, Cambridge, MA, USA. For protein exchange experiments, wells were precoated for $1 \mathrm{~h}$ with proteins which were found to delay the cell adhesion to TCPS. ${ }^{4}$ Human serum albumin (Alb, 20\% solution ( $\mathrm{w} / \mathrm{v})$ ) and immunoglobulin $\mathrm{G}(\mathrm{IgG}, 6 \%$ solution $(\mathrm{w} / \mathrm{v})$ ) were obtained from CLB. High-density lipoprotein (HDL, $100 \mu \mathrm{g} / \mathrm{mL}$ medium M 199), was a gift of Dr. V. W. M. van Hinsbergh, Gaubius Institute, Leiden, The Netherlands. HDL was obtained from a single male blood donor and dialyzed twice against phosphate buffered saline (PBS, obtained from NPBI, Emmer-Compascuum, the Netherlands) and medium M199 (from Gibco Biocult Co., Paisley, U.K.). The ratio of the volume of the protein solution $(\mathrm{mL})$, used for precoating, to the apparent TCPS surface area $\left(\mathrm{cm}^{2}\right)$ was 0.2 .

Harvesting of cells for experiments was performed by trypsinization (0.05\% trypsin $/ 0.02 \%$ EDTA). Trypsin was inactivated with CMS, when HEC were subsequently seeded onto TCPS in the presence of CMS. However, when HEC were to be seeded in culture medium containing $20 \%$ Fn-depleted serum (CMS-Fn), trypsin was first inactivated with CMS-Fn. Fn was removed from pooled serum by Gelatin-Sepharose affinity chromatography. ${ }^{8}$ The ratio of the seeding volume to the surface area ratio was 0.2 and seeding densities of $0.25,0.5,1.0,2.0,4.0,6.0$, and $8.0 \times 10^{4}$ cells $/ \mathrm{cm}^{2}$ were used.

At the end of the appropriate incubation time ( $30 \mathrm{~min}$ to $48 \mathrm{~h}$ ), adherent HEC were rinsed with phosphate buffered saline (PBS, obtained from NPBI, Emmer-Compascuum, The Netherlands) and lysed.

Cell lysis was performed for $10 \mathrm{~min}$ after the addition of $0.1 \mathrm{mNH}_{4} \mathrm{OH}$ (Merck, Darmstadt, FRG). ${ }^{9}$ Thereafter the adsorbed protein layer was rinsed three times with PBS, fixed with methanol for $15 \mathrm{~min}$ and rinsed again with PBS. The test wells, filled with PBS, were stored at $4^{\circ} \mathrm{C}$ before the measurement of protein adsorption.

\section{Adsorption and deposition of protein}

The experiments from which the results are shown in Figure 1 were started at the same time. This was also the case for the experiments presented in Figure 2. Each mark in the time courses of Figure 1 and Figure 2 represents an average value which has been obtained from protein deposition experiments carried out in three different wells of a 24-well TCPS cluster. Protein adsorption/deposition experiments presented in Figure 3 were carried out simultaneously; this was also the case for the experiments 
of Figure 4. Each lane in Figure 3 and Figure 4 represents an average value resulting from three experiments.

\section{Detection of adsorbed/deposited protein}

The deposition of protein was studied by means of a two step enzymeimmuno-assay (EIA) which permits the selective detection of adsorbed proteins. ${ }^{10,11}$ The EIA was carried out in the wells of the TCPS clusters as described in detail before, ${ }^{4}$ either directly after the exposure to protein solutions or culture medium, or after the ammonia/methanol treatment and PBS-storage following the exposure to these solutions or to a cell suspension in culture medium.

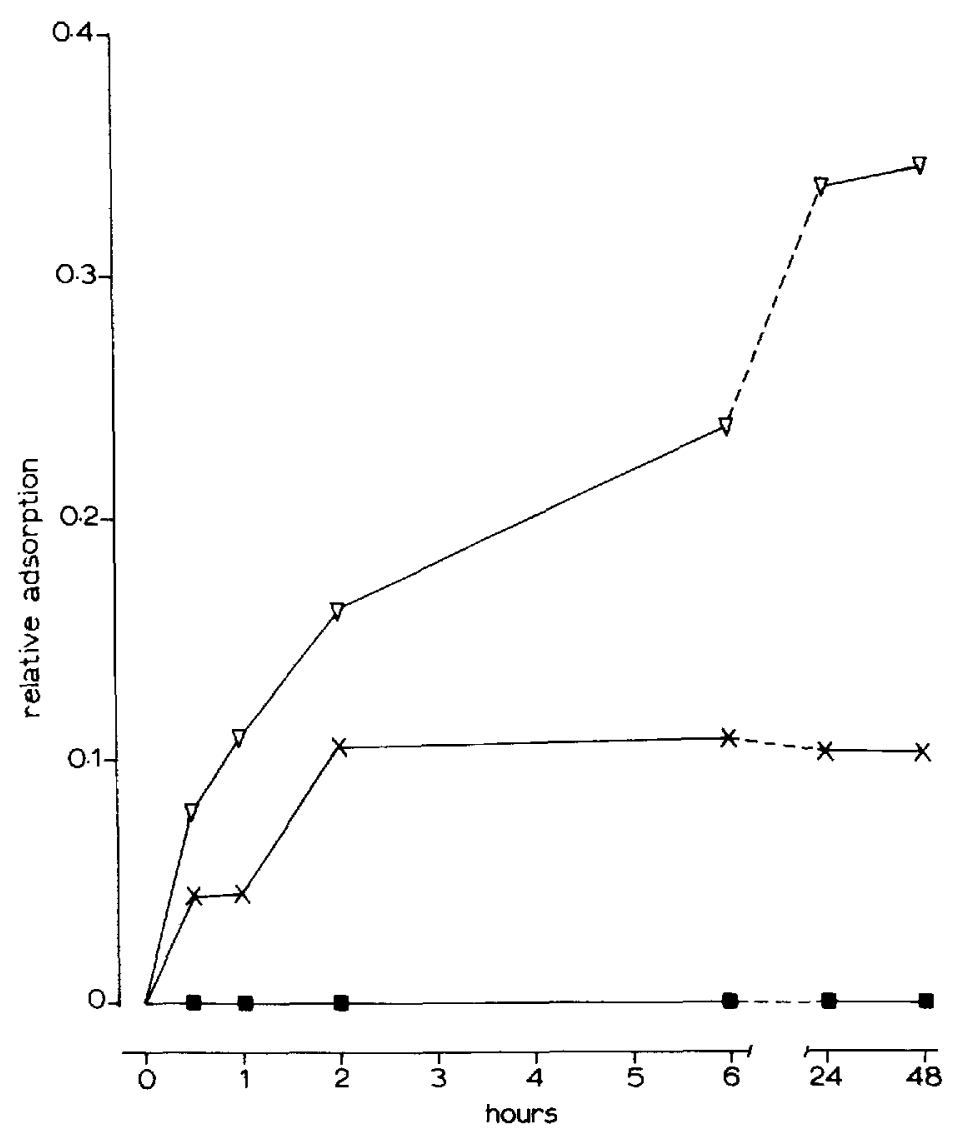

Figure 1. (c)Fn adsorption to TCPS from CMS $(\times-\times)$, CMS-Fn (-- - ) or HEC in CMS $(\nabla-\nabla)$ as measured with mEIA. $4 \times 10^{4}$ cells $/ \mathrm{cm}^{2}$ were seeded onto TCPS and after appropriate incubation times lysed with ammonia. The adsorbed protein layer was then fixated with methanol. The ammonial methanol treatment was also carried out with adsorbed protein layers in the absence of HEC. 


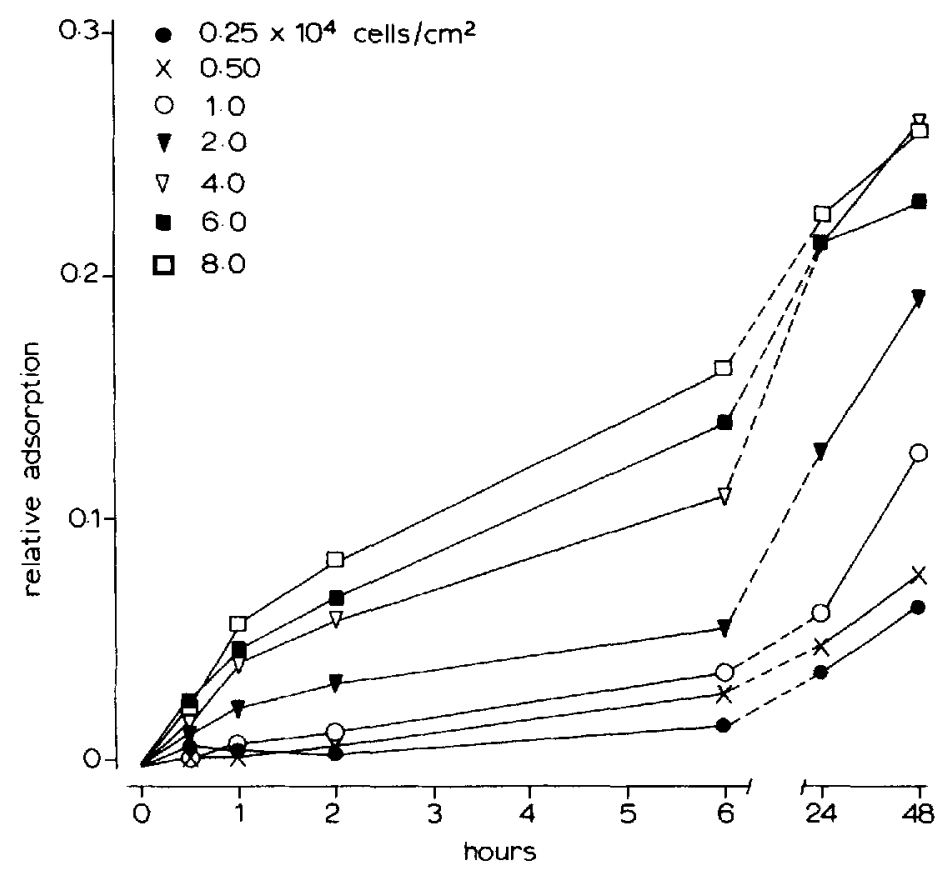

Figure 2. CFn adsorption onto TCPS, as measured with mEIA. Different densities of HEC were seeded in CMS-Fn. After appropriate incubation times, HEC were lysed with ammonia and the adsorbed protein layer was fixated with methanol.

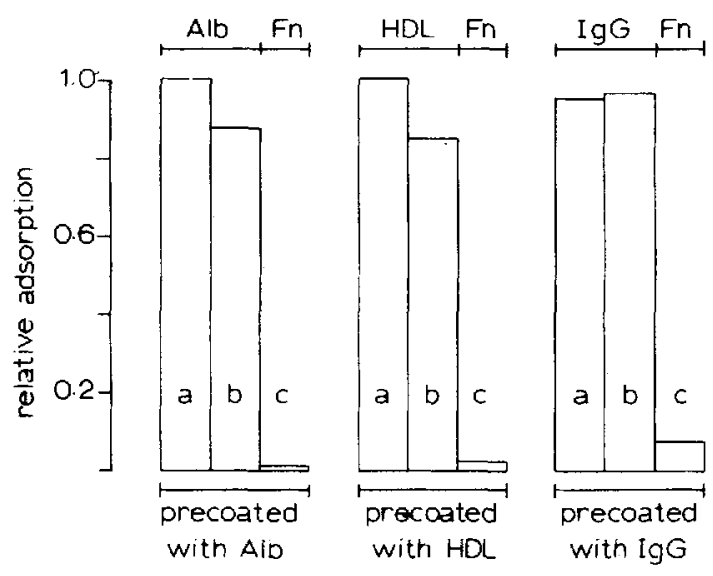

Figure 3. The relative adsorption onto TCPS of Alb, HDL or IgG after a 1-h precoat procedure (a), followed by a 6-h incubation period with CMS (b). The third lane (c) represents the Fn adsorption after the 6-h incubation period with CMS onto preadsorbed TCPS. Protein adsorption was measured with pEIA. Standard deviations of up to 0.1 were measured.

The relative protein adsorption data, presented in the figures are deduced from absorbances of a generated dye in the EIA (see below). These data are not directly related to amounts of protein in $\mu \mathrm{g} / \mathrm{cm}^{2}$. 

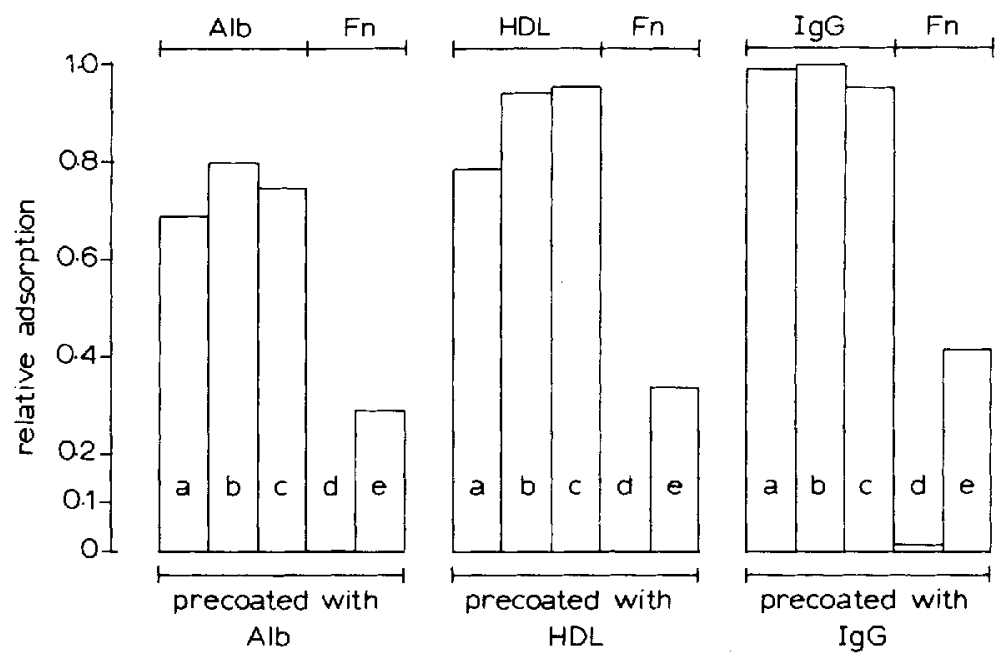

Figure 4. The relative adsorption onto TCPS of ALB, HDL, or IgG after a 1-h precoat procedure (a), followed by a 6-h incubation period with CMS-Fn (b) or a 6 -h incubation period with $4 \times 10^{4} \mathrm{HEC} / \mathrm{cm}^{2}$ in CMS-Fn (c). The Fn adsorption occurring from CMS-Fn is presented in lane d, while lane e represents Fn-deposition by HEC in CMS-Fn.

All adsorbed protein layers were treated with ammonia and methanol and measured with pEIA. Standard deviations of up to 0.08 were measured.

Briefly, for each well the following assay procedure was used. ${ }^{4}$ First, the test surface of each well was rinsed three times with washing buffer. This washing procedure was also carried out after each subsequent step. Then $200 \mu \mathrm{L} / \mathrm{cm}^{2}$ of first antibody solution was added (contact time $1 \mathrm{~h}$ ). After the washing procedure, $200 \mu \mathrm{L} / \mathrm{cm}^{2}$ of enzyme-labeled second antibody solution was added (contact time $1 \mathrm{~h}$ ). The enzymatic (color) reaction started after rinsing and the addition of $200 \mu \mathrm{L} / \mathrm{cm}^{2}$ of substrate- and leuko dye solution. This reaction was carried out in the dark during $30 \mathrm{~min}$ and was terminated by the addition of $100 \mu \mathrm{L} / \mathrm{cm}^{2}$ of $2 \mathrm{M}$ sulfuric acid. Part of the dye solution $(250 \mu \mathrm{L})$ was pipetted into a well of a 96 -well microtiter plate (A/S Nunc, Roskilde, Denmark) and the absorbance was measured at $450 \mathrm{~nm}$ with a multiscanner (Reader Micro Elisa System, Organon Teknika, Turnhout, Belgium).

The first antibody solution contained either polyclonal (pEIA) or monoclonal (mEIA) antibodies. The composition of first antibody solution and enzyme labeled second antibody solution for pEIA have been reported earlier. ${ }^{4}$

Mouse monoclonal antibody CLB-HEC-Fn 140 directed against human Fn (obtained from CLB) ${ }^{12}$ was purified from ascites by protein A Sepharose chromatography ${ }^{13}$ and stored as a solution of $0.5 \mu \mathrm{g} / \mathrm{mL}$. Before use in mEIA, it was diluted 100-fold with first antibody buffer. ${ }^{4}$ The enzyme labeled second antibody solution for mEIA also differed from the one used in pEIA. This solution contained goat-anti-mouse IgG (GMIgG) and horse radish peroxidase-labeled GMIgG (GMIgG ${ }^{\mathrm{HRP}}$ ). GMIgG and $\mathrm{GMIgG}^{\mathrm{HRP}}$ were 
obtained from CLB. GMIgG ${ }^{\mathrm{HRP}}$ was diluted 20 times with GMIgG, followed by 10,000-fold dilution with conjugate buffer.

\section{RESULTS}

\section{Deposition of (c)Fn onto TCPS}

HEC were seeded in culture medium containing 20\% serum (CMS) and Fn adsorption was measured after cell lysis by ammonia and fixation of the adsorbed protein layer by methanol.

The relative adsorption of Fn onto TCPS during $48 \mathrm{~h}$ after the seeding of $4 \times 10^{4} \mathrm{HEC} / \mathrm{cm}^{2}$ in CMS, was similar when measured with $\mathrm{pEIA}$ or mEIA (Fig. 1, pEIA not shown). The relative adsorption of Fn from CMS (without HEC) onto TCPS was also measured after ammonia/methanol treatment and carried out with mEIA (Fig. 1). The adsorption of Fn from CMS increased during the first $2 \mathrm{~h}$ and thereafter reached a plateau value for the next $48 \mathrm{~h}$. No adsorbed Fn was detected after exposure of TCPS to culture medium in which Fn-depleted serum was present (CMS-Fn) (Fig. 1). The cell adhesion and spreading rate during $6 \mathrm{~h}$ after seeding of $4 \times 10^{4} \mathrm{HEC} / \mathrm{cm}^{2}$ were the same, whether cells were seeded in CMS or in CMS-Fn. No difference in adhesion and spreading was observed by light microscope when higher or lower seeding densities were used.

HEC were seeded at different densities in the presence of CMS-Fn and cell lysis was carried out after various incubation times (Fig. 2). The relative adsorption of Fn onto TCPS, as measured with mEIA, increased both with increasing cell density and with exposure time.

\section{Exchange of preadsorbed proteins for Fn}

In an earlier study similar numbers of HEC were shown to adhere at $6 \mathrm{~h}$ onto TCPS, whether or not TCPS was previously precoated with proteins which inhibit HEC adhesion. ${ }^{4}$ Therefore, a 6-h incubation period of precoated TCPS with CMS, CMS-Fn or HEC in CMS-Fn was chosen. First the exchange of preadsorbed Alb, HDL or IgG for CMS-derived Fn was examined. In Figure 3 the relative adsorptions, as measured with pEIA, are shown. The relative amounts of adsorbed Alb, HDL, and IgG were about the same, when measured immediately after the precoating procedure (lane a). The same values were found after $6 \mathrm{~h}$ of incubation with CMS (lane b). No or hardly any serum-Fn was detected after incubation with CMS irrespective of the type of protein used for precoating (lane $c$ ).

The adsorption of cellular Fn onto TCPS was examined after $4 \times 10^{4}$ $\mathrm{HEC} / \mathrm{cm}^{2}$ were seeded onto TCPS precoated with Alb, HDL, or IgG (Fig. 4). The experiments were carried out in the presence of CMS-Fn. Cells were lysed by ammonia in order to expose the adsorbed protein layer which was then fixed by methanol. The controls (Fig. 4, lanes a, b, c, d) were also treated with ammonia/methanol. No significant differences in relative pro- 
tein adsorptions with or without the ammonia/methanol treatment were found (not shown). The relative amounts of adsorbed protein were about the same and varied between 0.7 and 1.0 when measured after $1 \mathrm{~h}$ of precoating (lane a), after the following $6 \mathrm{~h}$ exposure to CMS-Fn (lane b) and after a 6-h incubation period with HEC in CMS-Fn (lane c). No Fn was detected after exposure of the protein precoated TCPS surfaces to CMS-Fn during $6 \mathrm{~h}$ (lane d). When HEC were seeded in this culture medium, adhesion and spreading occurred as reported before ${ }^{4}$ and $\mathrm{Fn}$ was detected in the adsorbed protein layer (lane e). A relative Fn-adsorption of 0.34 was found for the HDL-precoated TCPS. Values of respectively 0.42 and 0.54 were found for the IgG-precoated and the Alb-precoated TCPS.

\section{DISCUSSION}

Human endothelial cells in culture are known to synthesize and secrete fibronectin (Fn) ${ }^{12,14-16} \mathrm{Fn}$ has also been detected in the extracellular matrix and in the subendothelium as a dense fibrillar network which mediates platelet adhesion. Clark et al. ${ }^{17}$ recently showed that cultured human endothelial cells grown from umbilical veins produce their own Fn for the in vitro attachment to collagen preadsorbed onto (unmodified) polystyrene.

This study demonstrates that cellular Fn (cFn) is deposited onto TCPS when endothelial cells from umbilical veins (HEC) are seeded in culture medium, which contains either normal serum or Fn-depleted serum. The amount of cFn depends on the number of seeded cells and on the time of incubation (Figs. 1, 2). Moreover, our results show that HEC immediately start with Fn-deposition after cell seeding, because cellular Fn was detected on the TCPS surface $30 \mathrm{~min}$ after HEC were seeded (Fig. 2). The conclusions with respect to the deposition of cFn onto TCPS are based on the results obtained with an enzyme immuno assay, in which we used a monoclonal antibody (mEIA). The advantage of the use of a monoclonal antibody as compared to a polyclonal antibody (pEIA), is the more specific detection of Fn. A possible disadvantage may be a decrease in sensitivity of protein detection if the antigenic determinant is involved in the adsorption onto the polymer surface. However, we did not observe major differences between the results obtained with the EIA, whether monoclonal or polyclonal antibodies were used. Both mEIA and pEIA do not yet allow a quantitative detection of adsorbed proteins. ${ }^{18}$

The study of Reinders et al. ${ }^{12}$ shows that cultured human vascular endothelial cells do not contain a storage site for $\mathrm{Fn}$. They conclude that the rate of Fn secretion depends solely on the rate of de novo protein synthesis. Therefore it is unlikely that Fn which has been detected on the TCPS surfaces originates from cytoplasma released during the lysis of HEC prior to the enzyme-immunoassay.

Using indirect immunofluorescence analysis to detect $\mathrm{cFn}$, Grinnell et al. ${ }^{19}$ have demonstrated that fibroblasts also deposit cFn onto tissue culture substrates (TCPS). Their data indicate that $\mathrm{cFn}$ is at first randomly present on the 
cell surface, next beneath the central portion of the cell and finally beneath the cell periphery. This transition coincides with the period during which cell spreading occurs. After longer time periods (2-8 h) a fibrillar pattern of cFn was observed. Grinnell et al. suggested that during initial attachment human fibroblasts deposit $\mathrm{CFn}$ onto TCPS which is required for their subsequent spreading.

In contrast to fibroblasts, HEC do not spread completely on TCPS in the absence of serum, as is shown by our present studies. In addition, no $\mathrm{cFn}$ was detected upon TCPS after $6 \mathrm{~h}$ when $8 \times 10^{4} \mathrm{HEC} / \mathrm{cm}^{2}$ were seeded under serum-free conditions (not shown). These preliminary results indicate that an increase in Fn-deposition upon TCPS is accompanied by an increase in spreading of HEC.

We did not investigate, whether the deposition of cFn onto uncoated TCPS occurs through an exchange of adsorbed proteins derived from CMS or from CMS-Fn. The possible exchange of adsorbed Alb, HDL, or IgG for (c)Fn was only examined after precoating of TCPS with these proteins.

Our results do not prove that an exchange of preadsorbed proteins for $\mathrm{cFn}$ occurs. After an exposure time of $6 \mathrm{~h}$ to culture medium (CMS), the amounts of preadsorbed Alb, HDL or IgG did not decrease as measured by the pEIA (Fig. 3). Moreover, no or hardly any serum-Fn could be detected on the precoated surfaces. Furthermore, we could not demonstrate that cellular-Fn replaced the preadsorbed proteins. Although adhering and spreading HEC deposit Fn onto the surfaces, the relative amounts of adsorbed Alb, HDL and IgG do not seem to decrease (Fig. 4).

The fact that exchange of preadsorbed $\mathrm{Alb}, \mathrm{HDL}$, or IgG for (c)Fn was not observed, may possibly be explained in two ways.

First, Fn derived from serum or from HEC, does not interact directly with TCPS, since complex formation between $\mathrm{Fn}$ and preadsorbed protein did occur without desorption of preadsorbed protein. Complex formation of Fn with collagen, fibrin and heparin is well known. ${ }^{20} \mathrm{Fn}$ is able to link cells with its cell binding site to collagen and fibrin. However data on the interaction of Fn with Alb, HDL, or IgG are lacking, although binding of another glycoprotein (a $43 \mathrm{~K}$ protein) to bovine serum albumin (BSA) has been reported. ${ }^{21}$ This $43 \mathrm{~K}$ protein is secreted by confluent and nonconfluent HEC as well as other cells. It is not identical to Fn, fragments of Fn, thrombospondin or $\alpha$-2-macroglobulin and it shows a high affinity to serum albumin. ${ }^{21}$

A second explanation is that desorption of Alb, HDL or IgG from precoated TCPS surfaces (Figs. 3,4) occurred, but that this was not detected by the applied immunoassay. In this immunoassay a compact monolayer of antibody molecules (first or second step of EIA) and not a compact monolayer of the adsorbed protein presumably defines the height of the adsorption plateau. In other words, the measurement of differences between various amounts of adsorbed protein is presumably not possible for high surface concentrations of protein. ${ }^{22}$ Therefore, if the amount of preadsorbed protein is not very much reduced during exposure to the culture medium (and cells), a decrease in protein adsorption will not be detected. 
However, an indication that preadsorbed Alb could have been exchanged for Fn, can be derived from experiments carried out by Grinnell. ${ }^{5} \mathrm{He}$ found that more serum-Fn out of the culture medium adsorbed onto TCPS which had been precoated with native (human) Alb, compared to TCPS which had been coated with denatured $\mathrm{Alb}$. We assume, that native $\mathrm{Alb}$ does not show large conformational changes during the adsorption onto the moderately wettable TCPS, and as a consequence, native Alb should be desorbed more easily than denatured Alb. Such a desorption is a condition for the exchange of surface bound Alb for Fn.

Our study shows, that adhering and spreading HEC deposit cFn onto TCPS, irrespective of the presence of a preadsorbed layer of proteins which delay cell adhesion.

\section{References}

1. P. B. van Wachem, T. Beugeling, J. Feijen, A. Bantjes, J. P. Detmers, and W. G. van Aken, "Interaction of cultured human endothelial cells with polymeric surfaces of different wettabilities," Biomaterials, 6, 403-408 (1985).

2. F. Grinnell, "The role of fibronectin in the bioreactivity of material surfaces," in Biocompatible Polymers, Metals and Composites, M. Szycher (Ed.), Technomic Publ. Co., Lancaster, PA, 1983, pp. 673-699.

3. J. Feijen, T. Beugeling, A. Bantjes, and C. Th. Smit Sibinga, "Biomaterials and interfacial pheomena," Adv. Cardiovasc. Phys., 3, 100 (1979).

4. P. B. van Wachem, C.M. Vreriks, T. Beugeling, J. Feijen, A. Bantjes, J. P. Detmers, and W. G. van Aken, "The influence of protein adsorption on interactions of cultured human endothelial cells with polymers," J. Biomed. Mater. Res., 21, 701-718 (1987).

5. F. Grinnell and M.K. Feld, "Adsorption characteristics of plasma fibronectin in relationship to biological activity," J. Biomed. Mater. Res., 15, 363-381 (1981).

6. E. A. Jaffe, R. L. Nachman, C. G. Becker, and C. R. Minick, "Culture of human endothelial cells derived from umbilical veins. Identification by morphologic and immunologic criteria," J. Clin. Invest., 52, 2745 (1973).

7. Ch. Willems, G. C. B. Astaldi, Ph. G. de Groot, M. C. Janssen, M. D. Gonsalves, W. P. Zeijlemaker, J. A. van Mourik, and W. G. van Aken, "Media conditioned by cultured vascular smooth muscle cells," Exp. Cell. Res., 139, 191 (1982).

8. E. Ruoslahti, E. G. Hayman, M. Piersbacher, and E. Engvall, "Fibronectin: purification, immunochemical properties and biological activity," Methods Enzymol., 82, 803-831 (1982).

9. K. S. Sakariassen, P. A. M. M. Aarts, Ph. G. de Groot, W. P. M. Houdijk, and J.J. Sixma, "A perfusion chamber developed to investigate platelet interaction in flowing blood with human vessel wall cells, their extracellular matrix and purified components," J. Lab. Clin. Med., 102, 522-535 (1983).

10. W. Breemhaar, D. L. Ellens, T. Beugeling, and A. Bantjes, "A novel application of a two step enzyme-immuno assay for the investigation of blood compatibility of materials," Life Support Systems, Proc. IXth Annual Meeting ESAO, W. B. Saunders Co., Eastbourne, U.K., 1982, pp. 295-300.

11. W. Breemhaar, E. Brinkman, D. J. Ellens, T. Beugeling, and A. Bantjes, "The interaction of polymers and blood plasma: preferential adsorption of high density lipoprotein from blood plasma onto biomaterial surfaces," Biomaterials, 5, 269-274 (1984). 
12. J. H. Reinders, P. G. de Groot, J. Dawes, N. R. Hunter, H. A. A. van Heugten, J. Zandbergen, M. D. Gonsalves, and J. A. van Mourik, "Comparison of secretion and subcellular localization of von Willebrand protein with that of thrombospondin and fibronectin in cultured human vascular endothelial cells," Biochim. Biophys. Acta, 844, 306-313 (1985).

13. J. W. Goding, "Conjugation of antibodies with fluorchromes: modification to the standard methods," J. Immunol. Methods, 12, 215 (1976).

14. E. A. Jaffe and D. F. Mosher, "Synthesis of fibronectin by cultured human endothelial cells," J. Exp. Med., 147, 1779-1791 (1978).

15. E. J. Mackarak, E. Kirby, T. Kirk, and N. A. Kefalides, Proc. Natl. Acad. Sci. USA, 75, 2621-2625 (1978).

16. C. R. Birdwell, D. Gospodarowicz, and G. L. Nicholson, Proc. Natl. Acad. Sci. USA, 75, 3273-3277, (1978).

17. R. A. F. Clark, J. M. Folkvord, and L. D. Nielsen, "Either exogenous or endogenous fibronectin can promote adherence of human endothelial cells," J. Cell. Sci., 82, 263-280 (1986).

18. W. Breemhaar, "An enzyme-immunoassay for the detection of blood components adsorbed to polymeric surfaces," Thesis, Twente University of Technology, Enschede, The Netherlands, October, 1985.

19. F. Grinnell and M. Feld, "Initial adhesion of human fibroblasts in serumfree medium: possible role of secreted fibronectin," Cell, 17, 117-129 (1979).

20. R. O. Hynes, "Fibronectins," Sci. Amer., 254 (6), 32-41 (1986).

21. H. Sage, C. Johnson, and P. Bornstein, "Characterization of a novel serum albumin-binding glycoprotein secreted by endothelial cells in culture," J. Biol. Chem., 259 (6), 3993-4007 (1984).

22. T. Beugeling, A. Poot, P. B. Van Wachem, E. Brinkman, W. G. Van Aken, and A. Bantjes, "Adsorption of plasma proteins and adhesion of cells onto polymer surfaces," $V^{\text {th }}$ Int. Conf. PIMS, September, 10-12, 1986.

Received March 20, 1987

Accepted May 27, 1987 